\title{
Laser, domains, and more: an interview with Shining Zhu
}

\author{
Guoqing Chang \\ Institute of Physics, Chinese Academy of Sciences, China
}

Guoqing Chang spoke to Shining Zhu of Nanjing University, about his insights into laser science, applications, and future prospects.

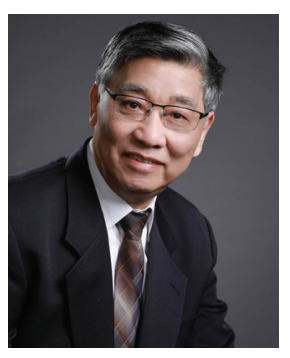

Shining Zhu
Chang: When did you first hear about laser? Zhu: As one of the greatest inventions of the 20th century, the laser has been applied to many fields, such as laser machining and processing, gravitational wave detection, precision metrology, laser spectroscopy and microscopy, 5Gl6G technology, microwave photonics, just to name a few. I cannot remember the exact time I heard about lasers, but I have definitely read articles about them in popular magazines ever since I was young. It was around that time when I learned about the Chinese scientists who had developed lasers shortly after Theodore Maiman. In college, I majored in condensed matter physics and took courses on optics, from which I learned more about lasers and became more interested in the topic. The real exposure to the laser came when I needed to use a laser to study phase transition in some crystal materials during my pursuit of a master's degree at Nanjing University. After earning the master's degree, I joined a research group led by Professor Naiben Ming to pursue a $\mathrm{PhD}$ degree. My PhD research focused on the development of new optical structures in crystals to manipulate and control laser light.

Chang: In your research, laser is an experimental tool for material characterization, right?

Zhu: This was the case at the beginning of my career. We started to take interest in the development of lasers as we discovered that nonlinear crystals with proper optical superlattice were suitable to achieve nonlinear frequency conversion. Such a capability allowed us to convert a laser's original wavelength to new wavelengths, even leading to a laser system that output three colors (red, green, and blue) simultaneously. In this scenario, quasi-white light was achieved. Later, we showed that the optical superlattice has unique advantages in the midinfrared (IR) wavelength range. Mid-IR lasers hold promise for many important applications (e.g., biomedicine, environmental monitoring, and national security), but mid-IR laser crystals are rare. Therefore, wavelength conversion becomes necessary to obtain mid-IR laser light from a source laser operating at the near-IR wavelength range. We developed a high-power, widely tunable mid-infrared laser system using a specially designed optical superlattice to perform wavelength conversion from a pump laser of $1.064 \mu \mathrm{m}$.

Chang: Your research on the Fibonacci optical superlattice connects the well-known golden ratio in mathematics and the quasi-phasematching in nonlinear optics. The results are really elegant. Can you give us more details?

(C) The Author. Published by SPIE and CLP under a Creative Commons Attribution 4.0 Unported License. Distribution or reproduction of this work in whole or in part requires full attribution of the original publication, including its DOI.. [DOI: 10.1117/1 .AP.2.5.050502]
Zhu: Before 1960, due to the lack of strong light sources, nonlinear optics mainly remained in sporadic theoretical research. After the emergence of the laser in 1960, nonlinear optics developed rapidly in both theory and experiment. In 1962, Bloembergen (Nobel Prize laureate in Physics in 1981) and his team theoretically proposed the concept of quasi-phase-matching (QPM) in nonlinear optics; that is, in order to enhance nonlinear optical processes, phase matching can be achieved by periodically changing the sign of the nonlinear coefficient of an optical crystal. In general, the modulation period in nonlinear crystals corresponding to the realization of OPM in the wavelength range from visible to IR was usually on the order of a few to a few tens of microns, which was extremely hard to implement.

The experimental verification of QPM had been hindered due to the difficulty of material fabrication, until the late 1970s when the research team in Nanjing University grew the first $\mathrm{LiNbO}_{3}(\mathrm{LN})$ crystals with periodic laminar ferroelectric domain. Naiben Ming and his colleagues discovered that such periodic laminar ferroelectric domain could be induced from the periodic growth striation in LN crystal, which could be controlled by use of the so-called Czochralski growth technology. Since the modulation of ferroelectric domains corresponds to the modulation of nonlinear coefficients in crystal, LN crystals with periodic laminar domain should be appropriate candidates for the study of QPM. After theoretical analysis and many attempts, they successfully grew LN crystal with the period at 6.8 microns, which met the QPM conditions for frequency doubling of laser light at $1064 \mathrm{~nm}$. Using this crystal, they produced bright green laser at $532 \mathrm{~nm}$, which robustly confirmed QPM principle. This work was first presented at the International Conference on Lasers held in Shanghai/Beijing in May of 1980, and then was immediately published in Applied Physics Letters.

Throughout the middle 1980s, because a variety of novel optical effects were discovered from the crystals with the periodic domains, the concept of optical superlattice was formally proposed as a new category of micro-structured materials. In 1982, Dan Shechtman (Nobel Prize laureate in Chemistry in 2011) discovered quasicrystal structure in aluminium-manganese alloy. The discovery was reported in Physical Review Letters in late 1984. Ming and his group began to consider whether the nonlinear crystals with quasi-periodic domain structure (i.e., the quasi-periodic optical superlattice) can be used to achieve QPM frequency conversion. They theoretically predicted that in a quasi-periodic superlattice two or more QPM optical parametric processes can be realized simultaneously. This is different from a periodic superlattice in which only a single QPM condition may be satisfied. Thus, they proposed the concept of the multiple quasi-phase-matching (MQPM) accordingly. This theoretic result was published in Physical Review B in 1990 - the year when I joined Ming's group as a $\mathrm{PhD}$ student.

As my thesis advisor, Ming asked me to experimentally fabricate such quasi-periodic structures that cannot be obtained using the previous crystal growth method for making periodic superlattice. After my efforts, I made progress in pattern poling $\mathrm{LN}$ and $\mathrm{LT}\left(\mathrm{LiTaO}_{3}\right)$ crystals by using the photolithography mask process. The quasi-periodic superlattices featuring a Fibonacci sequence were precisely fabricated to simultaneously meet the QPM condition for multiple wavelengthconversions. Using a picosecond optical parametric oscillator to provide wavelength-tunable laser light as the pump, frequency doubling 
using such a LT crystal with Fibonacci superlattice allowed us to achieve efficient second-harmonic generation (SHG) at multiple wavelengths. This work was later published in Physical Review Letters in 1997. In this study, the quasi-periodic superlattice took the Fibonacci sequence (i.e., golden ratio) because the sequence corresponds to the pentagonal symmetry in two- and three-dimensional quasicrystals.

Chang: How did the work move to generate higher-order nonlinear processes in a crystal?

Zhu: The previous work was to achieve a quasi-periodic superlattice, which can obtain high-efficiency SHG at multiple wavelengths. We wondered if it was possible to use one crystal to simultaneously generate SHG and third-harmonic generation (THG), or even other higherorder nonlinear processes. According to MQPM principle, we designed and prepared such a quasi-periodic superlattice crystal in which both the QPM conditions required for SHG and sum-frequency generation (SFG) can be met, thus allowing these two nonlinear processes to occur simultaneously. The cascade of SHG and SFG can effectively produce THG and this cascaded second-order nonlinear process corresponds to much higher conversion efficiency compared to THG from pure thirdorder nonlinearity. We submitted the manuscript to Science and surprisingly it was quickly accepted.

Chang: Communication between the Chinese and American communities became active after the 1980s. You visited Pennsylvania State University in the 1990s, right?

Zhu: I was a visitor at the Material Research Lab (MRL) of Pennsylvania State University from 1996 to 1998 . Their research on ferroelectric materials was second to none in the world, focusing on the piezoelectric properties of crystals and ceramics for sonar applications. In contrast, our superlattice research studied the domain structure in ferroelectric crystals targeting the applications in nonlinear optics. We learned from each other and realized that there are two types of domain structuresaverage domain structure and geometric domain structure-in ferroelectric and piezoelectric crystals. The material properties can be greatly improved, enhanced, and even designed by engineering the domain configurations in these crystals.

Chang: Through your narration, it seems that the imperfections in the crystal growing process resulted in unexpected periodic striation, which in turn led to the subsequent research in superlattice and QPM. Would you like to comment?

Zhu: Many scientific discoveries originated from unexpected experimental phenomena, including Shechtman's discovery of quasicrystals while studying aluminum-manganese alloys. This happened several times in my research group. For example, a graduate student did not find the expected laser light at the output end of a nonlinear crystal with specially designed superlattice structure; instead, he observed that some laser light appeared from the side surface at a special angle. After careful study, we found that this unexpected phenomenon corresponds to nonlinear Cherenkov radiation, which in fact represented the first observation of Cherenkov radiation in a nonlinear optical superlattice. The results were later published in the Physical Review Letters and the article was featured as the journal cover.

In another experiment, a student found that in addition to the round spot in the middle of the laser beam at the crystal output, rings of light appeared unexpectedly. After repeated experiments, we found that these rings came from elastic scattering caused by defects in the crystal. The superlattice structure of the crystal enhanced the scattering during the nonlinear optical process. It was extremely difficult to directly measure the elastic scattering inside a crystal. Our research provided a new method to characterize its spatial distribution in the crystal by measuring the angle, diameter, and intensity of these light rings.

It is quite common that new materials and novel structural designs are often accompanied by new discoveries and may even produce new physical concepts. This kind of unexpected discovery makes us feel that research, though sometimes exhausting, is a lot of fun.

Chang: What research fields are you currently focusing on?

Zhu: Quantum information processing must be integrated on a chip to be practical, and therefore we are developing various technologies to enable large-scale integrated quantum photonics. We are the first to develop quantum chips based on superlattices. With the development of 5Gl6G communications, we are also working hard to apply integration technology to microwave photonics, laser frequency combs, quantum simulations, etc. In addition, we utilize metasurfaces and artificial intelligence to develop new imaging technologies. For example, we successfully developed the first broadband achromatic metalens and the first $60 \times 60$ optical field imaging meta-lens array. We also published the results of a cascaded waveguide array in Advanced Photonics (doi: 10.1117/1.AP.2.3.036001). Our most recent work-which was published in Science-demonstrated a 100-path parametric downconversion photon-pair source by integrating a metalens-array with a BBO nonlinear crystal, which shows promise for high-dimensional entanglement and multiphoton-state generation.

In the future, we will continue to use microstructures to engineer the optical properties of materials and fabricate functional devices to control the amplitude, phase, polarization, and angular momentum of a laser beam. I firmly believe that microstructure science holds promise for many prospective applications.

Shining Zhu is a professor at the School of Physics and a principal investigator $(\mathrm{PI})$ at the National Laboratory of Solid State Microstructures, Nanjing University, China. He is also an academician of the Chinese Academy of Sciences, and a fellow of OSA, COS, and APS. He got his master's and PhD degrees from the Physics Department of Nanjing University. Since then he has been working on functionally microstructured materials, paying close attention to their applications in laser, nonlinear, and quantum optics. He and his collaborators have published more than 500 papers in $\mathrm{SCl}$ journals, including Science, Nature, Physical Review Letters, Nature Materials, Nature Photonics, etc. Due to his outstanding contributions, he and his colleagues received the First-Class State Natural Science Award in 2006. He was appointed as dean of the School of Physics in Nanjing University from 2005 to 2011 and also served as founding dean of a newly established College of Engineering and Applied Science at Nanjing University from 2009 to 2012.

Guoqing Chang graduated with both bachelor's and master's degrees from the Electronics Engineering Department of Tsinghua University. He obtained his $\mathrm{PhD}$ degree in electrical engineering from the Center for Ultrafast Optical Science at the University of Michigan. After staying at the University of Michigan as a postdoctoral research fellow for about one year, he joined the Research Laboratory of Electronics at Massachusetts Institute of Technology as a postdoctoral research associate. In August 2012, he moved to the Center for Free Electron Laser (CFEL) at Hamburg (Germany) as the head of the Helmholtz Young investigator group "Ultrafast Laser Optics and Coherent Microscopy" under the ultrafast optics and x-ray division. He was granted tenure in 2016. In 2017, he joined Institute of Physics, Chinese Academy of Sciences, as a professor. His current research focuses on high-power ultrafast fiber lasers, ultrafast nonlinear optics, and multi-photon microscopy for biomedical imaging. 